This item was submitted to Loughborough's Research Repository by the author.

Items in Figshare are protected by copyright, with all rights reserved, unless otherwise indicated.

\title{
Decision-making after the product-service shift and some implications for
} ergonomics

PLEASE CITE THE PUBLISHED VERSION

PUBLISHER

(c) Taylor \& Francis

VERSION

AM (Accepted Manuscript)

LICENCE

CC BY-NC-ND 4.0

\section{REPOSITORY RECORD}

Molloy, Ella-Mae, Carys E. Siemieniuch, and Murray A. Sinclair. 2019. "Decision-making After the Productservice Shift and Some Implications for Ergonomics”. figshare. https://hdl.handle.net/2134/9281. 
This item was submitted to Loughborough's Institutional Repository (https://dspace.lboro.ac.uk/) by the author and is made available under the following Creative Commons Licence conditions.

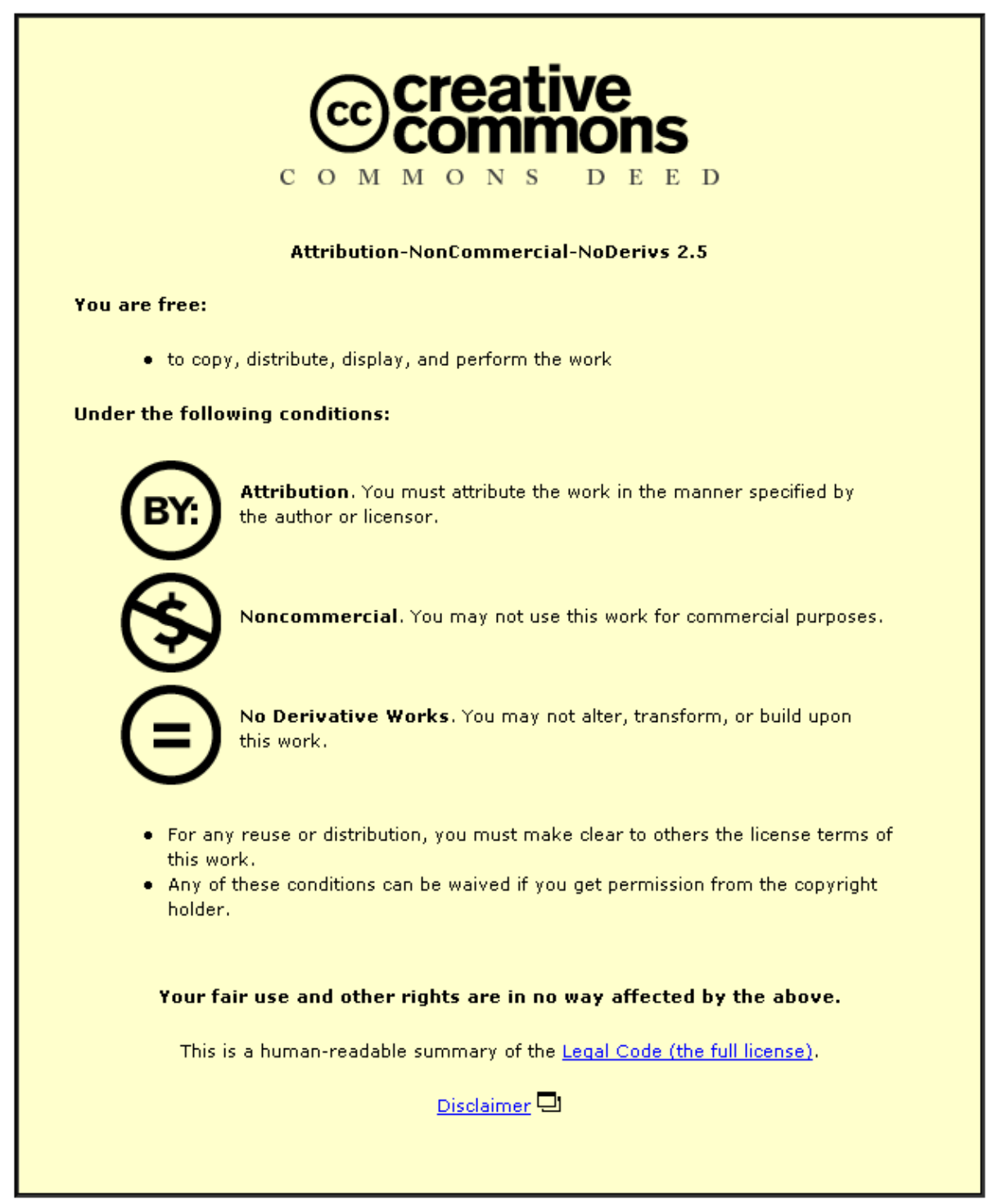

For the full text of this licence, please go to: http://creativecommons.org/licenses/by-nc-nd/2.5/ 


\title{
DECISION-MAKING AFTER THE PRODUCT- SERVICE SHIFT AND SOME IMPLICATIONS FOR ERGONOMICS
}

\author{
E.M. Molloy. M.A. Sinclair, C.E. Siemieniuch
}

Loughborough University

This paper introduces a Decision-Making System Framework developed during research in a UK (EPSRC) funded Grand Challenge, entitled 'Knowledge and Information Management - through life' (KIM). The development of the framework is outlined, along with a brief outline of the validation work..

The future use of the framework will be discussed, with reminders that the goal of this framework is not to provide the 'right' answer, which is improbable, if not impossible, to predict when dealing with such extended and complex systems

\section{Introduction}

A significant challenge, particularly for engineering organisations, is the 'productservice shift', whereby organisations move from delivering a product to the provision of through-life, sustained capability support (Oliva and Kallenberg 2003; Brady, Davies et al. 2005). This affects companies in both the internal organisation and in the processes and procedures they employ; particularly in the area of knowledge and information management. This is the focus of the KIM Grand Challenge.

Furthermore, the problems will get steadily worse as systems evolve from standalone status to components of systems-of-systems, with the issues associated with this shift. Whilst technology and technical infrastructures play an important role within the whole area of knowledge and information management, the making of complex decisions is still, essentially, a human and organisational issue. This is the context of this paper.

The paper outlines the background of complexity and decision-making systems (DMS) before outlining the development and subsequent validation of a DMF (Decision Making system Framework) and supporting process. It builds on work initially presented at the INCOSE Conference in Boston, 2007 (Molloy, Siemieniuch et al. 2007).

\section{The Impact of Complexity}

What confounds the whole area under discussion is the issue of complexity: "Complexity is really just reality without the simplifying assumptions that we make in order to understand it.” (Allen, Boulton et al. 2005). This is a neat encapsulation, but for the purposes of this research, the definition of complexity is that provided by Rycroft and Kash (Rycroft and Kash 1999) - "A system exhibits complexity if it is composed of many integrated entities of heterogeneous parts, which act in a coordinated way and whose behaviour is typically nonlinear."

Issues of complexity and emergence are often considered from two perspectives. Firstly, there is the emergence of order from disorder. Conway's 'Game of Life' is an 
example of this [(Gardner 1970). A different approach, attuned to an organisational perspective, is to consider the emergence of a different order (or disorder) from an established or planned order, and this is the perspective for this paper.

In this latter class of complexity, it is also possible to identify two sources of complexity (Baccarini 1996) - intrinsic and induced complexity, outlined below.

\section{Intrinsic complexity}

Intrinsic complexity is inherent in the problem: it arises from the interactions between components in a system, both intended and unintended. Whilst it must be accepted that intrinsic complexity cannot be totally eliminated, there are ways to reduce it, for example:

- Modularity and maturity in design can contain complexity,

- Architectures for core components and rigid adherence to standards,

- Clear understanding of the problem context,

- $\quad$ A stable project environment (budget, timescales, client coherence, partners etc.)

This last point is the one with the biggest impact, but the most difficult to achieve.

\section{Induced Complexity}

Induced complexity arises from how the organisation chooses to structure itself and its project teams to address the problem of intrinsic complexity.

Inducing extra complexity into the delivered capability via inappropriate project management is discussed in the literature (deMeyer, Loch et al. 2002; Williams 2005). The style of project management is the result of organisational culture, role assignment and knowledge of the problem area (Sinclair 2007); however, the client, the legal framework and many other entities can create a project environment near certain to trigger problems of induced complexity and, as with intrinsic complexity, the emergence of undesirable, inappropriate behaviour of the system.

\section{Emergent Behaviour}

Emergent behaviour can be defined as follows: “...however the system was designed to behave, it behaves differently, often unexpectedly, and not necessarily in desirable ways." (Sinclair 2007). Part of the solution to unwanted emergent behaviour arises by reconfiguring decision support to help cope with complexity and the surprises such emergent behaviour can bring - systems ergonomics issues are critical in this.

\section{Decision-making systems and decision support systems}

Decision-making is affected by external environmental and commercial pressures, and by internal effects and pressures. Many of these, such as established processes, organisational structures, and incentivisation policies, are determined by the company's overall strategy. It is important that strategy is developed and implemented in an arena of good governance, otherwise the endeavour to make good decisions will be vitiated and detrimental emergent behaviour will be a near-certainty.

\section{Decision-Making Systems (DMS)}

Included within the term DMS as it is used within this paper are:

- $\quad$ Agents - software or human based, who are involved with decisions

- Activities - the decision-making activities which enable decisions to be made 
- Infrastructure and technology - to facilitate decision-making, including computer based support

- Knowledge and Information. Its distribution across the organization (the 'knowledge configuration') determines the effectiveness of decisions made.

DMS will of course be affected by time (for example, time available in which to make the decision, time by when the decision must be made, time when the effect of the decision is realized and expected duration of the decision) and the style of decisionmaking.

\section{Decision Support Systems (DSS)}

In this research, DSS are considered to form part of the overall DMS. In much of the literature (Finlay 1989; Silver 1991)DSS are considered to include only computer based tools. However, this research widens that definition to include any form of support or guidance, which may be computer based or from a more human source, such as Communities of Practice (CoP) (Wenger, McDermott et al. 2004)

\section{Development of a decision-making systems framework}

The aim of the DMS framework and the associated process of use is to assist organisations in configuring their DMS to help them to cope with the risks and opportunities of long life, complex, engineered projects and systems. A top level view of the framework is shown in figure 1. The framework has been developed from metaanalysis of accident and incident reports, pilot studies and industrial based case studies, all of which embrace complexity in some way. These are outlined below.

\section{Literature studies}

The incidents meta-analysed were predominantly accident cases where a wealth of information and documentation is available. These studies investigated the decision processes and issues leading up to the various incidents, not the causes nor the blame. The analyses identified commonalities between the incidents; for example, people not available to do the work, poor communication, complacency, etc. A categorization of these issues and commonalities allowed initial identification of the attributes of DMS (e.g. agents, activities, infrastructure, knowledge and information) and the organisational aspects which could be affected by the DMS (e.g. internal and external variables, cultures and level of decision-making) and provided the development of the initial framework.

\section{Pilot studies}

A number of pilot studies were carried out, to aid development of the framework and to develop methodology for the subsequent studies. Two student-based studies were investigated. Both were on design projects that mimic many of the organisational and technical aspects of full-scale engineering projects. These were followed by two field studies, one on the design and build of an innovative educational building, and an engineering study, looking at enterprise modeling in a research and engineering setting.

All of the studies looked at the identification of key decision points and the mechanisms of decision-making and decision support, leading to further development of the framework (and associated system) along with some key initial findings, such as: (1) not all decisions are consciously made, creating a rich environment for miscommunication and emergent behaviour; (2) corporate and top level strategy is not 
always flowed down or instantiated in day to day activities, which can impact on decision rationale; and (3) small scale organisational success with stakeholder involvement does not always scale up to extensive success and can have unexpected outcomes, meaning the right processes may not work as expected if applied for misguided reasons.

\section{Case Studies}

This research involves two industrial case studies, in addition to the pilot studies outlined above.

In both the studies, a qualitative approach to data gathering was used. Preliminary work involved analysis of existing documentation followed by a combination of email communication, interviews and workshops. The studies took place within a multinational aerospace organization experiencing the issues involved in the product-service transition. They focused on the critical process of deciding whether or not to bid for a contract, exploring identified problems at different levels of activity decomposition. Data was gathered on activities and roles, information flows and decision points, along with process and problem perception and other contextual details. All of these are exemplars of induced complexity.

An outline of these studies is included in the table below.

\begin{tabular}{|c|c|c|}
\hline Study & Problem statement & Overview of key findings \\
\hline $\begin{array}{l}\text { Industrial } \\
\text { study } 1\end{array}$ & $\begin{array}{l}\text { A stage of the lifecycle was perceived } \\
\text { to have become congested with } \\
\text { mandated activities and reviews, } \\
\text { leaving little time to complete all } \\
\text { necessary work to progress the bid. }\end{array}$ & $\begin{array}{l}\text { A change of focus has occurred with } \\
\text { contracts written on service based instances } \\
\text { and a more delicate balance of capability } \\
\text { and profit. For the bid process, this } \\
\text { represents new risk and the analysis is not } \\
\text { as mature as it could be to deal with this. } \\
\text { The move outlined above has also } \\
\text { encouraged overlap in review activities } \\
\text { between different functions } \\
\text { It is not enough to adapt existing processes } \\
\text { to deal with these new contracts - new } \\
\text { process must be developed and employed } \\
\text { Not all decisions are consciously made, } \\
\text { especially during status review. }\end{array}$ \\
\hline $\begin{array}{l}\text { Industry } \\
\text { study } 2\end{array}$ & $\begin{array}{l}\text { There was concern about the quality } \\
\text { and consistency of one of the } \\
\text { technical reviews, which takes place } \\
\text { pre-bid. It was perceived that there } \\
\text { was a lack of understanding of the } \\
\text { training requirements for those } \\
\text { involved with the review; one } \\
\text { obvious knock-on effect being an } \\
\text { incorrect assessment of the technical } \\
\text { issues in bids. This could be very } \\
\text { fortunate in 'bet the company' bids. }\end{array}$ & $\begin{array}{l}\text { Confusion arises when progressing a service } \\
\text { based contract through the auspices of a } \\
\text { technical review with regards to what } \\
\text { should be reviewed and a concern that some } \\
\text { things are not reviewed at all, leading to } \\
\text { unknown risk. } \\
\text { Purpose and importance of activities is not } \\
\text { clear, which has issues for tailoring and } \\
\text { consistency of output. } \\
\text { These things need to be addressed before } \\
\text { appropriate training can be developed. }\end{array}$ \\
\hline
\end{tabular}

\section{The DMS framework}

A framework has been constructed based on the review of accident reports and the case studies, as outlined in the previous section This framework allows data to be captured and represented in a common format. A representation is given in Figure 1. The row headings are component parts of a DMS. The column headings are aspects of the 
overall system or system of systems, which could affect or be affected by the DMS. These are outlined below.

\section{Components of the DMS}

The following are the constituent parts of a DMS:

- Agents: DM (decision-making) agents may be human or software based, for example, who are involved in the DM process.

- Activities: DM activities are those activities necessary for a decision to be made.

- Infrastructure and technology: That which provides support for the DM process.

- Knowledge and information: DM knowledge and information is that which is

\begin{tabular}{|c|c|}
\hline Feature of DMS & Issue \\
\hline \multirow{3}{*}{ A. Agents/ Roles } & Poor role/ agent definition \\
\hline & Poor role/ agent allocation \\
\hline & Non-availability of roles/ agents \\
\hline \multirow{2}{*}{ B. Activities } & Inappropriate activities \\
\hline & $\begin{array}{l}\text { Poor definition of activities (unclear } \\
\text { or fuzzy boundaries) }\end{array}$ \\
\hline \multirow{2}{*}{$\begin{array}{l}\text { C. Infrastructure } \\
\text { and Technology }\end{array}$} & Inappropriate infrastructure \\
\hline & Non-availability of infrastructure \\
\hline \multirow{2}{*}{$\begin{array}{l}\text { D. Knowledge } \\
\text { and Information }\end{array}$} & $\begin{array}{l}\text { Inappropriate knowledge and/ or } \\
\text { information }\end{array}$ \\
\hline & $\begin{array}{c}\text { Non-availability of knowledge and/ or } \\
\text { information }\end{array}$ \\
\hline
\end{tabular}

Figure 1 Top-level view of the framework

necessary for a decision to be made.

The DM knowledge and information flows through the infrastructure and technology, around the DM activities to the agents to allow decisions to be made.

\section{Impacting variables on the DMS}

These include:

- Internal variables: Contextual issues (e.g. stage of lifecycle)

- Environmental variables: External influences (e.g. legislation, partners)

- Organisational variables: Structure, empowerment, risk -taking, etc.

- Level of DM: Strategic, tactical or operational. 


\section{Using the DMS Framework}

The aim of the DMS is to assist the audit of any DMS within an organization and to aid its dynamic reconfiguration for the extended lifecycles of the product-service shift.

Figure 1 is a top-level view of the prototype DMS Framework. Issues identified in an audit may be oriented in this framework, according to the component of the DMS and impacting variable concerned. Mapping of issues allows 'quick-view' comparisons of both clusters and of voids to highlight areas for investigation. This surfaces issues and allows stakeholders to commence discussions to enact improvements; it is essentially a tool to initiate and direct discussion. It does not present ready-made solutions.

Work is in progress on the application and use of the framework and its databases. Detailed use scenarios are being developed. It is currently envisaged that application will be possible both for prospective and retrospective work.

On the negative side, there are some issues that the framework does not currently address. These include training and workload. However, as noted above, the framework has been used to investigate problems in these areas. Furthermore, the use of the DMS framework and associated processes relies heavily on honest identification of issues and subsequent categorization. Given that few people are able to understand more than a small part of their own organization, and given the power relationships and politics to be found in any organization, these are not easy goals to achieve.

Finally, an upgrading process will be made available for the framework, to allow it to be tailored for a given organization and its ever-changing context and to provide updates as new information becomes available.

\section{Validation}

A final case study was used as validation for this work. The aim was to validate both the tool and the process for use.

The study took place in a manufacturing organisation, a different industry to that which formed the focus of the primary studies for this work.

During the time of the study, the organisation was experiencing a transition to lean manufacturing techniques (Womack and Jones 1994).

Some of the problems they were experiencing included:

- Management of a transient workforce

- Loss of knowledge through loss of key personnel

- Issues arising from changing customer requirements.

The DMS process was used alongside more established techniques (such as the Role Matrix Technique, IDEF activity modeling, CIM-OSA) and has been shown to compliment such techniques, providing extra contextual information from a decision making point of view and helping to identify some potential route causes for issues ascertained elsewhere.

The study highlighted the level of knowledge currently required to use the prototype version of the tool. A more usable form will need to be developed before widespread use.

\section{Conclusion}

The framework does not provide 'right' answers, but informs organisations about the characteristics of their DMS to enable them to be resilient in unexpected situations.

With new contract and project types, there is a requirement for new processes, which may require more (different) people and resources and will require wider knowledge. 
These processes must be accepted and integrated into the organisational processes if they are to be effective. Considerations from agility show that a paradigm shift is required. This includes the hard bits of the organization to change - culture, trust, leadership etc.

There will also need to be a significant upgrading of the infrastructure that joins the community of organizations and people that deliver and use the capability. This must at least accompany, if not be in the lead, of the changes outlined above.

With the growth of 'through-life capability' contracts comes a shift in responsibility. Suppliers' responsibility to provide a through-life service means the decisions they make are now long-term. This calls for better decision support, with better identification and assessment of risk (technical and business). These form part of the new processes and must be easy to use, if not implicit within regular working practices. If a decision is not consciously made, the outcome is neither known nor accounted for. This can lead to unanticipated behaviour (emergence), increasing risks and missed opportunities.

With this interlinking of issues, a multi-disciplinary approach is a necessity in any such contract. The collaboration requirements mean that whole supply chains must work in partnership. The multi-disciplinary approach will cross organisational boundaries, calling for a better alignment between different organisational processes, as noted in the industrial studies. Without full information flows, trust and partnerships are essential.

By focusing attention away from the facility at the point of handover to the services supported by that facility over the longer term, the current changeover to product-service projects provides new challenges for decision-making.

With service typically being consumed or used as it is produced, it is probable that each instantiation will be different. It becomes fundamental for a service organization to ensure that it is resilient enough to cope with a very changeable environment.

All of the above emphasises the key message of this paper: effective and appropriate, embedded decision support is essential for successful service organizations, and tools for this task must be flexible enough to withstand the manifold challenges of complexity

\section{Acknowledgements}

The work presented herein was undertaken in the Knowledge and Information Management (KIM) Through-Life Grand Challenge Project (www.kimproject.org) funded primarily by the Engineering and Physical Sciences Research Council (EPSRC Grant Number EP/C534220/1), the Economic and Social Research Council (ESRC Grant Number RES-331-27-0006.

\section{References}

Allen, P. M., J. Boulton, et al. (2005). The implications of complexity for business process and strategy. Managing organisational complexity: philosophy, theory and application. K. Richardson. Mansfield, MA, ISCE Publishing: 397-418.

Baccarini, D. (1996). "The concept of project complexity: a review." International Journal of Project Management 14: 201-204.

Brady, T., A. Davies, et al. (2005). "Creating value by delivering integrated solutions." International Journal of Project Management 23: 360-365.

deMeyer, A., C. H. Loch, et al. (2002). "Managing project uncertainty: from variation to chaos." Sloan Management Review 43(2): 60-67.

Finlay, P. (1989). Introducing decision support systems. Oxford, NCC Education 
Services.

Gardner, M. (1970). "The fantastic combinations of John Conway's new solitaire game "life"." Scientific American 223(October): 120-123.

Molloy, E.-M., C. E. Siemieniuch, et al. (2007). The Effects of Complexity on the Product-Service Shift. International Conference on Complex Systems. Boston, MA.

Oliva, R. and R. Kallenberg (2003). "Managing the transtion from products to services." International Journal of Service Industry Management 14(2): 160-172.

Rycroft, R. W. and D. E. Kash (1999). The complexity challenge. London, Pinter.

Silver, M. S. (1991). Systems that support decision makers: description and analysis. Chichester, John Wiley \& Sons.

Sinclair, M. A. (2007). "Ergonomics issues in future systems." Ergonomics 50(12): 1957 - 1986.

Wenger, E., R. McDermott, et al. (2004). Cultivating communities of practice, Harvard Business School Publications.

Williams, T. M. (2005). "Assessing and moving on from the dominant project management discourse in the light of project over-runs." IEEE Transactions on Engineering Management 52(4): 497-508.

Womack, J. P. and D. T. Jones (1994). "From lean production to the lean enterprise." Harvard Business Review 72(1): 93-103. 